\title{
Effects of dietary probiotic, liquid feed and nutritional concentration on the growth performance, nutrient digestibility and fecal score of weaning piglets
}

\author{
Song Zhang ${ }^{1,2}$, Dong Huy Yoo ${ }^{1,3}$, Xiang $\mathrm{Ao}^{1}$, and In Ho Kim ${ }^{1, *}$
}

* Corresponding Author: In Ho Kim Tel: +82-41-550-3652, Fax: +82-41-565-2949,

E-mail: inhokim@dankook.ac.kr

' Department of Animal Resource and Science, Dankook University, Cheonan 31116, Korea

${ }^{2}$ Kemin Industries (China) Co., Ltd. Sanzao, Zhuhai 519040, China

${ }^{3}$ All The Best Co., Ltd. Seoul 05399, Korea

ORCID

Song Zhang

https://orcid.org/0000-0003-0570-1909

Dong Huy Yoo

https://orcid.org/0000-0001-7179-5291

Xiang Ao

https://orcid.org/0000-0001-8851-7706

In Ho Kim

https://orcid.org/0000-0002-8785-6201

Submitted Jun 8, 2019; Revised Aug 27, 2019; Accepted Oct 30, 2019
Objective: This study was conducted to investigate the effects of dietary probiotic blend and liquid feed program at different nutritional densities on growth performance, nutrient digestibility, fecal score of weaning piglets.

Methods: A total of 120 weaning pigs with an initial body weight of $7.05 \pm 0.93 \mathrm{~kg}$ per pig (21 days of age) were randomly allocated into 1 of the following 8 dietary treatments ( 3 replicates per treatment with 5 pigs per replicate) in a $2 \times 2 \times 2$ factorial arrangement (nutrition levels: apparent metabolic energy $[\mathrm{AME}]=3,500 \mathrm{kcal} / \mathrm{kg}$, crude protein $[\mathrm{CP}]=20 \%$ vs AME $=3,400 \mathrm{kcal} / \mathrm{kg}, \mathrm{CP}=19.42 \%$; feed types:dry vs wet; probiotics levels: $0 \mathrm{mg} / \mathrm{kg}$ vs $300 \mathrm{mg} / \mathrm{kg}$ ). Results: During d 5 to $\mathrm{d} 15$, greater average daily gain (ADG) and average daily feed intake $(A D F I)(p<0.05)$ were observed in probiotics treatments. During d 15 to $d 25$, gain:feed (G:F) ratio $(\mathrm{p}<0.05)$ were significantly improved in probiotics, wet feed and high nutrition diet. Moreover, two interactions i) between nutrition levels and feed types, and ii) between nutrition levels and probiotics were found in G:F ratio. Furthermore, there was a significant positive interaction on G:F among those 3 factors $(p<0.05)$. Overall, increasing ADG, ADFI, and G:F ratio were detected in probiotics treatment significantly $(\mathrm{p}<0.05)$. Besides, an obvious reduction on fecal score was observed in probiotics treatment from $\mathrm{d} 0$ to $\mathrm{d} 5(\mathrm{p}<0.05)$. There was an interactive effect on fecal score between feed types and nutrition concentrations from $\mathrm{d} 5$ to $\mathrm{d} 25(\mathrm{p}<0.05)$.

Conclusion: These results indicated that probiotics supplementation could benefit growth performance and reduce the frequency of watery feces. Besides, wet feed program (feed:water $=1: 1.25$ ) could improve the G:F. The effect of liquid feed or probiotic could be influenced by dietary nutrition density in weaned piglets. An increased value of G:F was obtained when wet feeding a high nutrition diet (100 kcal higher than NRC 2012 recommendations) was supplemented with probiotics for 15 to 25 days.

Keywords: Dietary Probiotic Blend; Liquid Feeding Program; Nutrition Levels; Growth Performance; Fecal Score; Nutrient Digestibility; Weaning Pigs

\section{INTRODUCTION}

Gastrointestinal disturbances immediately post weaning can cause heavy economic loss in the pig industry. The weaning transition is a complex period during which the piglets have to cope with abrupt separation from their mothers and adapt to new environment where they are mixed with other litters. In addition, their diet will be switched from highlydigestible (liquid) milk to a less digestible and more-complex solid feed during this transition. Weaned at an early age ( 21 to $35 \mathrm{~d}$ ) in intensive production systems has probably exacerbated the level of general stress in these immature animals [1].

In the past decade, various nutritional methods or solutions to minimize the weaning 
losses have been tested, some of which have been widely implemented in practice. Probiotics, which is a modulator to increase many active behaviors, plays an important role in gut-brain axis regulation [2]. Many references demonstrate different probiotics capacities can enhance productivity in weaning piglets and increase gain:feed (G:F) ratio [3], average daily gain (ADG) [4] and nutrient digestibility [5]. Direct action of the probiotics can achieve a higher bioavailability of feed nutrients, indirect gut health modulation (relieving weaning stress, preventing diarrhea, improving the intestinal microbiota profile, etc.) or perhaps a combination of both may be involved [6]. Liquid feed diets have been widely used in western and southern Europe for 20 years, especially in France and Italy [7]. There has been an increase in the use of fermented liquid and liquid feed in the European Union (EU) since the ban on the use of antibiotics as growth promoters in pig feed [8]. Piglets grew faster on the liquid diet due to a higher feed intake (FI), a better transition from milk feeding and lower risk of dehydration [9]. With the advancement of additives and feeding technology, probiotics and liquid feeding would be used in most swine farms. However, what nutritionists always focus on is the formulation designs. Various nutrition levels and the liquid feed program might influence the effects of additives in pigs. Therefore, we hypothesized there might be an interaction among nutrition levels, the liquid feeding program and probiotics. However, no research has been carried out to determine the interaction among probiotics, the liquid feeding program and nutrition designs. Consequently, the objective of the study is to determine effects of dietary probiotic blend and liquid feed program at different nutritional densities on growth performance, nutrient digestibility, fecal score in weaning piglets.

\section{MATERIALS AND METHODS}

The protocols used for the current experiment were approved by the Animal Care and Use Committee of Dankook University, Korea.

\section{Animal, diet, experimental design}

A total of 120 weaning pigs (21 days of age) with an initial body weight (BW) of $7.05 \pm 0.93 \mathrm{~kg}$ per pig were randomly allocated into 1 of 8 dietary treatments ( 3 replicates per treatment with 5 pigs per replicate) in a $2 \times 2 \times 2$ factorial arrangement with 2 levels of nutrition density apparent metabolic energy $(\mathrm{AME})=14.63 \mathrm{~kJ} / \mathrm{kg}$ or $3,500 \mathrm{kcal} / \mathrm{kg}, \mathrm{CP}=20 \%$ vs $\mathrm{AME}=$ $14.23 \mathrm{~kJ} / \mathrm{kg}$ or $3,400 \mathrm{kcal} / \mathrm{kg}, \mathrm{CP}=19.42 \%), 2$ types of feed (dry vs wet), the liquid feed was prepared by mixing meal and water in a 1:2.5 ratio. The 2 levels of probiotics $(0 \mathrm{mg} / \mathrm{kg}$ vs $300 \mathrm{mg} / \mathrm{kg}$ ) were provided by Daehan feed mill company (Bacillus subtilis $2 \times 10^{8}$ colony-forming unit [CFU]/g, Entero- coccus faecium $2 \times 10^{8} \mathrm{CFU} / \mathrm{g}$ ). The 8 experimental diets were: TRT1, high nutrition $\times$ dry type without probiotics; TRT2, high nutrition $\times$ dry type with probiotics; TRT3, high nutrition $\times$ wet type without probiotics; TRT4, high nutrition $\times$ wet type with probiotics; TRT5, low nutrition $\times$ dry type without probiotics; TRT6, low nutrition $\times$ dry type with probiotics; TRT7, low nutrition $\times$ wet type without probiotics; TRT8, low nutrition $\times$ wet type with probiotics. This research was divided into three periods: Phase 1, d 0 to 5; Phase 2, d 5 to 15; Phase 3 , d 15 to 25 . All diets were formulated to contain approximately equal amounts of essential amino acids and the amino acid was on standard ileal digestibility basis. Ca, absorbable $\mathrm{P}$, and $\mathrm{Na}$, based on the analytical data from the feedstuffs. Different nutrition concentration diets meet or exceed the requirements suggested by the National Research Council (NRC [10]) (Table 1).

Chromic oxide $\left(\mathrm{Cr}_{2} \mathrm{O}_{3}\right)$ was added to each diet at $0.2 \%$ as an indigestible marker to evaluate the nutrient digestibility on d 5, d 15, and d 25. Pigs were housed in an environmentally controlled nursery facility with slatted plastic flooring and a mechanical ventilation system. The temperature of the room was maintained at approximately $30^{\circ} \mathrm{C}$ for the first week of the experiment, after which it was reduced by $1^{\circ} \mathrm{C}$ per week over the next 4 weeks. Each pen $(1.8 \times 1.0 \mathrm{~m})$ was equipped with a self-feeder and a nipple waterer to allow ad libitum access to feed and water throughout the experimental period.

\section{Sampling and measurements}

The individual pig BW and feed consumption from each pen was monitored to calculate the ADG, average daily feed intake (ADFI) and G:F ratio on $0, d 5, d$, and d 25. Fecal samples were collected from two pigs in each pen (1 gilt and 1 barrow) on the $\mathrm{d} 5, \mathrm{~d} 15$, and $\mathrm{d} 25$ of the experiment to determine the apparent total tract digestibility of dry matter (DM), N, and energy.

Feed samples were dried at $70^{\circ} \mathrm{C}$ for $72 \mathrm{~h}$, after which they were ground to pass through a $1-\mathrm{mm}$ screen. The DM and $\mathrm{N}$ concentrations were determined according to the AOAC [11]. Representative samples of each ingredient were hydrolyzed for $23 \mathrm{~h}$ at $1101^{\circ} \mathrm{C}$ with or without performic acid oxidation for Cys and Met, and other AA, respectively, and AA was separated by ion exchange chromatography and quantified by photometric detection after ninhydrin reaction European Commission [12].

The fecal score was determined by the average value of five pigs of each pen by using a 5-grade score system [13]. The standard of this system is as following: $1=$ hard, dry pellets in a small, hard mass; 2 = hard, formed stool that remains firm and soft; 3 = soft, formed and moist stool that retains its shape; $4=$ soft, unformed stool that assumes the shape of the container; $5=$ watery, liquid stool that can be poured. Scores were recorded on a pen basis following observations 
Table 1. Diet compositions (as fed basis)

\begin{tabular}{|c|c|c|}
\hline Item & $\begin{array}{l}\text { High nutrition } \\
\text { diet }\end{array}$ & $\begin{array}{c}\text { Low nutrition } \\
\text { diet }\end{array}$ \\
\hline Yellow corn & 33.32 & 36.95 \\
\hline Extruded corn & 20.00 & 20.00 \\
\hline Whey powder (78\%) & 7.00 & 7.00 \\
\hline Soybean meal (46\%) & 8.28 & 5.98 \\
\hline Fermented soybean meal & 5.00 & 5.00 \\
\hline Extruded soybean meal & 5.00 & 5.00 \\
\hline Skimmed milk powder & 7.00 & 7.00 \\
\hline Fish meal & 3.00 & 3.00 \\
\hline Sugar & 2.00 & 2.00 \\
\hline Glucose & 2.00 & 2.00 \\
\hline SDPP & 3.00 & 3.00 \\
\hline Soy oil & 1.33 & 0.00 \\
\hline Limestone & 0.55 & 0.55 \\
\hline MCP & 0.68 & 0.72 \\
\hline Salt & 0.10 & 0.10 \\
\hline Lysine-HCl $(98.5 \%)$ & 0.44 & 0.42 \\
\hline DL-methionine (99\%) & 0.30 & 0.29 \\
\hline L-threonine (98.5\%) & 0.20 & 0.19 \\
\hline L-tryptophan (10\%) & 0.30 & 0.30 \\
\hline Choline (50\%) & 0.10 & 0.10 \\
\hline Vitamin premix ${ }^{1)}$ & 0.20 & 0.20 \\
\hline Mineral premix ${ }^{2)}$ & 0.20 & 0.20 \\
\hline Total & 100.0 & 100.0 \\
\hline \multicolumn{3}{|c|}{ Calculated composition ${ }^{3)}(\%)$} \\
\hline $\mathrm{CP}$ & 20.00 & 19.42 \\
\hline Crude fat & 4.70 & 3.42 \\
\hline Ash & 5.49 & 6.05 \\
\hline AME (kcal/kg) & 3500 & 3400 \\
\hline $\mathrm{Ca}$ & 0.7 & 0.7 \\
\hline AP & 0.5 & 0.5 \\
\hline Lys & 1.58 & 1.55 \\
\hline \multicolumn{3}{|c|}{ Apparent ileal digestible amino acid } \\
\hline SID-Lys & 1.48 & 1.45 \\
\hline $\mathrm{ME} / \mathrm{CP}$ & 175 & 175 \\
\hline CP/SID-LyS & 13.79 & 13.79 \\
\hline SID-TSAA/SID-Lys & 0.60 & 0.60 \\
\hline SID-Thr/SID-Lys & 0.62 & 0.62 \\
\hline SID-Trp/SID-Lys & 0.17 & 0.17 \\
\hline
\end{tabular}

SDPP, spray-dried porcine plasma; MCP, monocalcium phosphate; AME, apparent metabolic energy; SID, standard ileal digestibility; CP, crude protein; TSAA, total sulfur amino acid.

1) Supplied per kg diet: 4,000 IU vitamin $A, 800$ IU vitamin $D_{3}, 171$ IU vitamin $\mathrm{E}, 2 \mathrm{mg}$ vitamin $\mathrm{K}, 4 \mathrm{mg}$ vitamin $\mathrm{B}_{2}, 1 \mathrm{mg}$ vitamin $\mathrm{B}_{6}, 16 \mu \mathrm{g}$ vitamin $\mathrm{B}_{12}, 11 \mathrm{mg}$ pantothenic acid, $20 \mathrm{mg}$ niacin and $0.08 \mathrm{mg}$ biotin.

2) Supplied per kg diet: 220 mg Cu, 175 mg Fe, 191 mg Zn, 89 mg Mn, 0.3 mg I, 0.5 $\mathrm{mg} \mathrm{Co}$ and $0.4 \mathrm{mg} \mathrm{Se}$.

${ }^{3)}$ Calculated values.

of individual pigs and signs of stool consistency in the pen.

\section{Statistical analyses}

Data were analyzed as a completely randomized block design, with a $2 \times 2 \times 2$ factorial arrangement, using general linear model procedure [14]. Variability in the data was expressed as the pooled standard error of mean, and $\mathrm{p}<0.05$ was considered statistically significant, whereas $\mathrm{p}<0.10$ was considered a tendency.

\section{RESULTS}

\section{Growth performance and nutrient digestibility of dry matter, energy and nitrogen}

The results of growth performance and nutrient digestibility of DM, energy and nitrogen are presented in Table 2, 3, respectively. In the phase $2, \mathrm{ADG}$ and ADFI were greater $\mathrm{p}<$ 0.05 ) in probiotics treatments comparing to the non-probiotics treatments. In the phase 3 , probiotics, wet feed and high nutrition diet significantly improved G:F $(\mathrm{p}<0.05)$. Besides, those pigs fed high nutrition diet appeared to have greater $A D G$ and $\mathrm{G}: \mathrm{F}$ than that fed low nutrition diet $(\mathrm{p}<0.05)$. Moreover, the interactive influence on G:F appeared not only between nutrition levels and feed types but also between nutrition levels and probiotics $(\mathrm{p}<0.05)$. Interestingly, there was a significant probiotics $\times$ feed type $\times$ nutrition density interaction on G:F $(\mathrm{p}<0.05)$. Piglets fed the diet containing probiotics had increased ADG, ADFI, and G:F comparing to that receiving the diet without probiotics $(\mathrm{p}<0.05)$. Besides, high nutrition diet significantly raised ADG and ADFI $(\mathrm{p}<0.05)$. No interactive response was found through the entire experiments $(p>0.05)$. There was no difference in nutrient digestibility of DM, energy and nitrogen as well ( $p>0.05)$.

\section{Fecal score}

The results of fecal score are appeared in Table 4. An obvious reduction on fecal score was observed in probiotics treatment from $\mathrm{d} 0$ to $\mathrm{d} 5(\mathrm{p}<0.05)$. There was an interactive effect on fecal score between feed types and nutrition concentrations from d 5 to d 25 ( $\mathrm{p}<0.05)$.

\section{DISCUSSION}

The results of this study showed that dietary probiotics blend can increase the ADG, ADFI, and G:F ratio throughout the trial. And the results are accordance with the previous paper of our laboratory [15], which evidenced positive effects on growth performance in the overall period associated with the inclusion of multi-strain probiotics (B. licheniformis and B. subtilis) in the diets. Generally, probiotics or probiotics mixtures could improve ADG [16-18] and increase G:F ratio [19, $20]$ in post-weaned piglets. However, the influence of probiotics on ADFI is inconsistent. In agreement with our results, Nguyen et al [21] documented that increasing the inclusion of the probiotics mixture (Bacillus coagulans, B. licheniformis, B. subtilis, and Clostridium butyricum) levels in the diets linearly increased the ADG and ADFI for day 0 to 7 as well as 
Table 2. Effects of feeding program on growth performance in weaning pigs

\begin{tabular}{|c|c|c|c|c|c|c|c|c|c|c|c|c|c|c|c|c|}
\hline \multirow{4}{*}{ Items } & TRT $1^{1)}$ & TRT2 $^{1)}$ & TRT3 $3^{1)}$ & TRT4 ${ }^{11}$ & TRT5 $^{1)}$ & TRT6 $^{1)}$ & TRT7 $^{1)}$ & TRT8 ${ }^{1)}$ & \multirow{4}{*}{ SEM } & \multicolumn{7}{|c|}{$p$-value ${ }^{2)}$} \\
\hline & \multicolumn{4}{|c|}{ High nutrition density } & \multicolumn{4}{|c|}{ Low nutrition density } & & \multirow{3}{*}{ Probiotics } & \multirow{3}{*}{$\begin{array}{l}\text { Feed } \\
\text { type }\end{array}$} & \multirow{3}{*}{$\begin{array}{c}\text { Nutrition } \\
\text { density }\end{array}$} & \multirow{3}{*}{$\begin{array}{l}\text { Probiotics } \\
\times \text { feed type }\end{array}$} & \multirow{3}{*}{$\begin{array}{c}\text { Feed type } \\
\times \text { nutrition } \\
\text { density }\end{array}$} & \multirow{3}{*}{$\begin{array}{l}\text { Probiotics } \\
\times \text { nutrition } \\
\text { density }\end{array}$} & \multirow{3}{*}{ Interactior } \\
\hline & \multicolumn{2}{|c|}{ Dry type } & \multicolumn{2}{|c|}{ Wet type } & \multicolumn{2}{|c|}{ Dry type } & \multicolumn{2}{|c|}{ Wet type } & & & & & & & & \\
\hline & $\mathrm{NC}$ & Probiotics & NC & Probiotics & NC & Probiotics & NC & Probiotics & & & & & & & & \\
\hline \multicolumn{17}{|c|}{ Body weight $(\mathrm{kg})$} \\
\hline Initial & 7.08 & 7.08 & 7.07 & 7.05 & 7.04 & 7.04 & 7.02 & 7.02 & 0.61 & 0.930 & 0.963 & 0.985 & 0.997 & 0.991 & 0.994 & 0.994 \\
\hline Phase $1^{3)}$ & 8.09 & 8.16 & 8.13 & 8.16 & 8.16 & 8.09 & 8.03 & 8.11 & 0.63 & 0.940 & 0.966 & 0.951 & 0.928 & 0.957 & 0.963 & 0.913 \\
\hline Phase2 ${ }^{3)}$ & 10.75 & 10.95 & 10.82 & 11.22 & 10.12 & 10.35 & 10.20 & 10.54 & 0.69 & 0.216 & 0.755 & 0.557 & 0.975 & 0.874 & 0.988 & 0.964 \\
\hline Phase $3^{3)}$ & 13.56 & 15.15 & 14.38 & 15.84 & 12.79 & 14.11 & 12.93 & 14.31 & 1.02 & 0.118 & 0.535 & 0.064 & 0.690 & 0.982 & 0.906 & 0.951 \\
\hline \multicolumn{17}{|l|}{ Phase1 } \\
\hline $\operatorname{ADG}(g)$ & 201 & 216 & 213 & 221 & 224 & 211 & 201 & 218 & 18 & 0.970 & 0.980 & 0.608 & 0.532 & 0.634 & 0.716 & 0.485 \\
\hline ADFI (g) & 212 & 217 & 239 & 237 & 234 & 233 & 219 & 239 & 20 & 0.739 & 0.515 & 0.697 & 0.336 & 0.818 & 0.765 & 0.631 \\
\hline $\mathrm{G}: \mathrm{F}$ & 0.968 & 0.998 & 0.889 & 0.930 & 0.960 & 0.907 & 0.917 & 0.916 & 0.032 & 0.366 & 0.063 & 0.864 & 0.234 & 0.495 & 0.197 & 0.663 \\
\hline \multicolumn{17}{|l|}{ Phase2 } \\
\hline$A D G(g)$ & 266 & 279 & 269 & 307 & 196 & 225 & 217 & 243 & 20 & $<0.001$ & 0.248 & 0.083 & 0.891 & 0.723 & 0.945 & 0.616 \\
\hline ADFI (g) & 332 & 349 & 314 & 424 & 266 & 315 & 290 & 329 & 24 & 0.005 & 0.183 & 0.106 & 0.768 & 0.238 & 0.560 & 0.145 \\
\hline$G: F$ & 0.800 & 0.802 & 0.859 & 0.723 & 0.738 & 0.727 & 0.751 & 0.738 & 0.046 & 0.099 & 0.986 & 0.242 & 0.739 & 0.300 & 0.415 & 0.313 \\
\hline \multicolumn{17}{|l|}{ Phase3 } \\
\hline ADG $(\mathrm{g})$ & 281 & 419 & 356 & 461 & 267 & 376 & 273 & 376 & 42 & 0.078 & 0.323 & 0.002 & 0.366 & 0.751 & 0.805 & 0.826 \\
\hline ADFI (g) & 418 & 623 & 529 & 669 & 452 & 551 & 403 & 565 & 61 & 0.141 & 0.486 & 0.003 & 0.283 & 0.986 & 0.634 & 0.470 \\
\hline $\mathrm{G}: \mathrm{F}$ & 0.668 & 0.674 & 0.672 & 0.690 & 0.587 & 0.684 & 0.676 & 0.666 & 0.010 & 0.005 & 0.006 & 0.001 & 0.093 & 0.004 & 0.042 & $<0.001$ \\
\hline \multicolumn{17}{|l|}{ Overall } \\
\hline $\operatorname{ADG}(\mathrm{g})$ & 259 & 323 & 292 & 352 & 230 & 283 & 236 & 291 & 21 & 0.007 & 0.220 & 0.002 & 0.448 & 0.978 & 0.815 & 0.918 \\
\hline ADFI (g) & 342 & 432 & 385 & 484 & 334 & 393 & 321 & 405 & 28 & 0.031 & 0.256 & $<0.001$ & 0.249 & 0.666 & 0.569 & 0.851 \\
\hline$G: F$ & 0.756 & 0.747 & 0.763 & 0.726 & 0.687 & 0.720 & 0.736 & 0.719 & 0.016 & 0.011 & 0.482 & 0.509 & 0.196 & 0.105 & 0.182 & 0.625 \\
\hline
\end{tabular}

SEM, pooled standard error of means; NC, negative control; ADG, average daily gain; ADFI, average daily feed intake; G:F, gain:feed.

1) TRT1, high nutrition density feed $\times$ dry type $\times$ none; TRT2, high nutrition density feed $\times$ dry type $\times$ probiotics; TRT3, high nutrition density feed $\times$ wet type $\times$ none; TRT4, high nutrition density feed $\times$ wet type $\times$ probiotics; TRT5, low nutrition density feed $\times$ dry type $\times$ none; TRT6, low nutrition density feed $\times$ dry type $\times$ probiotics; TRT7, low nutrition density feed $\times$ wet type $\times$ none; TRT8, low nutrition density feed $\times$ wet type $x$ probiotics.

2) $p<0.05$ was considered statistically significant, whereas $p<0.10$ was considered a tendency.

3) Phase 1, 0 to 5 days; Phase 2, 5 to 15 days; Phase 3, 15 to 25 days.

ADG for day 8 to 21. However, Zhao et al [22] believed probiotics (Lactobacillus reuteri and Lactobacillus plantarum) blend could not affect ADFI. Different probiotics strains and concentration of probiotics might be an important factor affecting the ADFI. Especially, since probiotics also live on nutrient, they might compete with host for nutrients in the diet or make hosts require more feed.

The key factor underlying the poor postweaning performance is the immediate reduction in FI due to the abrupt transition from liquid milk to less digestible feeds [23,24],

Table 3. Effects of feeding program on digestibility in weaning pigs

\begin{tabular}{|c|c|c|c|c|c|c|c|c|c|c|c|c|c|c|c|c|}
\hline \multirow{4}{*}{ Items (\%) } & TRT1 $^{1)}$ & TRT2 $^{1)}$ & TRT3 $^{1)}$ & TRT4 $^{1)}$ & TRT5 $^{1)}$ & TRT6 $^{1)}$ & TRT7 $^{1)}$ & TRT8 ${ }^{1)}$ & \multirow{4}{*}{ SEM } & \multicolumn{7}{|c|}{$p$-value ${ }^{2)}$} \\
\hline & \multicolumn{4}{|c|}{ High nutrition density } & \multicolumn{4}{|c|}{ Low nutrition density } & & \multirow{3}{*}{ Probiotics } & \multirow{3}{*}{$\begin{array}{l}\text { Feed } \\
\text { type }\end{array}$} & \multirow{3}{*}{$\begin{array}{c}\text { Nutrition } \\
\text { density }\end{array}$} & \multirow{3}{*}{$\begin{array}{c}\text { Probiotics } \times \\
\text { feed type }\end{array}$} & \multirow{3}{*}{$\begin{array}{c}\text { Feed type } \\
\times \text { nutrition } \\
\text { density }\end{array}$} & \multirow{3}{*}{$\begin{array}{c}\text { Probiotics } \\
\times \text { nutrition } \\
\text { density }\end{array}$} & \multirow{3}{*}{ Interaction } \\
\hline & \multicolumn{2}{|c|}{ Dry type } & \multicolumn{2}{|c|}{ Wet type } & \multicolumn{2}{|c|}{ Dry type } & \multicolumn{2}{|c|}{ Wet type } & & & & & & & & \\
\hline & NC & Probiotics & NC & Probiotics & NC & Probiotics & NC & Probiotics & & & & & & & & \\
\hline \multicolumn{17}{|l|}{$5 d$} \\
\hline Dry matter & 83.31 & 84.21 & 84.38 & 84.77 & 84.21 & 84.25 & 83.50 & 83.97 & 0.53 & 0.245 & 0.315 & 0.341 & 0.637 & 0.276 & 0.168 & 0.750 \\
\hline Nitrogen & 82.64 & 83.23 & 83.81 & 82.58 & 83.03 & 83.65 & 83.64 & 83.69 & 0.91 & 0.156 & 0.267 & 0.543 & 0.104 & 0.739 & 0.624 & 0.448 \\
\hline Energy & 83.86 & 84.35 & 84.75 & 85.36 & 84.68 & 83.79 & 85.31 & 85.22 & 0.77 & 0.265 & 0.543 & 0.438 & 0.681 & 0.963 & 0.624 & 0.541 \\
\hline \multicolumn{17}{|l|}{$10 d$} \\
\hline Dry matter & 84.50 & 84.93 & 83.51 & 85.06 & 84.44 & 83.79 & 85.04 & 83.89 & 0.95 & 0.854 & 0.453 & 0.631 & 0.360 & 0.357 & 0.400 & 0.509 \\
\hline Nitrogen & 83.57 & 82.65 & 83.62 & 84.31 & 83.65 & 84.31 & 82.86 & 83.06 & 0.59 & 0.804 & 0.654 & 0.265 & 0.664 & 0.631 & 0.817 & 0.168 \\
\hline Energy & 83.83 & 84.35 & 84.75 & 85.36 & 84.32 & 85.28 & 83.31 & 85.16 & 0.80 & 0.647 & 0.169 & 0.735 & 0.261 & 0.736 & 0.547 & 0.440 \\
\hline \multicolumn{17}{|l|}{$25 d$} \\
\hline Dry matter & 84.34 & 85.94 & 85.08 & 86.89 & 84.32 & 84.78 & 85.43 & 84.66 & 0.69 & 0.122 & 0.174 & 0.116 & 0.725 & 0.599 & 0.060 & 0.458 \\
\hline Nitrogen & 82.61 & 84.85 & 83.83 & 85.05 & 82.97 & 83.41 & 83.60 & 83.41 & 0.78 & 0.187 & 0.361 & 0.099 & 0.724 & 0.458 & 0.154 & 0.863 \\
\hline Energy & 83.97 & 84.80 & 84.45 & 86.01 & 82.84 & 84.10 & 84.42 & 83.83 & 0.80 & 0.078 & 0.189 & 0.180 & 0.863 & 0.620 & 0.448 & 0.257 \\
\hline
\end{tabular}

SEM, pooled Standard error of means; NC, negative control.

1) TRT1, high nutrition density feed $x$ dry type $\times$ none; TRT2, high nutrition density feed $\times$ dry type $\times$ probiotics; TRT3, high nutrition density feed $\times$ wet type $\times$ none; TRT4, high nutrition density feed $\times$ wet type $\times$ probiotics; TRT5, low nutrition density feed $\times$ dry type $\times$ none; TRT6, low nutrition density feed $\times$ dry type $\times$ probiotics; TRT7, low nutrition density feed $\times$ wet type $\times$ none; TRT8, low nutrition density feed $\times$ wet type $\times$ probiotics.

2) $p<0.05$ was considered statistically significant, whereas $p<0.10$ was considered a tendency. 
Table 4. Effects of feeding program on fecal score in weaning pigs

\begin{tabular}{|c|c|c|c|c|c|c|c|c|c|c|c|c|c|c|c|c|}
\hline \multirow{4}{*}{ Items } & TRT11) & TRT2 $2^{1)}$ & TRT3 $^{1)}$ & TRT4 ${ }^{1)}$ & TRT5 $^{1)}$ & TRT6 $^{1)}$ & TRT7 $7^{1)}$ & TRT8 $^{11}$ & \multirow{4}{*}{ SEM } & \multicolumn{7}{|c|}{ p-value ${ }^{2)}$} \\
\hline & \multicolumn{4}{|c|}{ High nutrition density } & \multicolumn{4}{|c|}{ Low nutrition density } & & \multirow{3}{*}{ Probiotics } & \multirow{3}{*}{$\begin{array}{l}\text { Feed } \\
\text { type }\end{array}$} & \multirow{3}{*}{$\begin{array}{c}\text { Nutrition } \\
\text { density }\end{array}$} & \multirow{3}{*}{$\begin{array}{c}\text { Probiotics } \\
\times \text { feed } \\
\text { type }\end{array}$} & \multirow{3}{*}{$\begin{array}{c}\text { Feed type } \\
\times \text { nutrition } \\
\text { density }\end{array}$} & \multirow{3}{*}{$\begin{array}{c}\text { Probiotics } \\
\times \text { nutrition } \\
\text { density }\end{array}$} & \multirow{3}{*}{ Interaction } \\
\hline & \multicolumn{2}{|c|}{ Dry type } & \multicolumn{2}{|c|}{ Wet type } & \multicolumn{2}{|c|}{ Dry type } & \multicolumn{2}{|c|}{ Wet type } & & & & & & & & \\
\hline & NC & Probiotics & NC & Probiotics & NC & Probiotics & NC & Probiotics & & & & & & & & \\
\hline \multicolumn{17}{|l|}{ Fecal score $e^{3)}$} \\
\hline $0-5 d$ & 2.5 & 2.9 & 2.7 & 2.8 & 2.9 & 2.8 & 2.9 & 3.0 & 0.09 & 0.039 & 0.229 & 0.099 & 0.624 & 1.000 & 0.063 & 0.229 \\
\hline $5-15 d$ & 3.1 & 2.9 & 2.9 & 3.0 & 3.1 & 3.0 & 3.0 & 3.0 & 0.06 & 0.476 & 0.290 & 0.476 & 0.720 & 0.021 & 0.476 & 0.290 \\
\hline $15-25 d$ & 3.2 & 3.0 & 3.0 & 3.2 & 3.3 & 3.0 & 3.0 & 3.1 & 0.08 & 0.650 & 0.184 & 0.650 & 0.650 & 0.003 & 0.880 & 0.650 \\
\hline
\end{tabular}

therefore, sufficient FI is a big challenge for subsequent growth performance. Previous studies indicated liquid feeding reconstituted to $13 \% \mathrm{DM}$ and fed via liquid feeders in the early weaning period improves FI which resulted in greater body weight gain $[25,26]$. Whereas, this study showed liquid feed which were reconstituted to $25 \%$ DM only enhanced G:F ratio. The difference might result from the different DM. Geary et al [27] reported DM content in the range of 255 to $149 \mathrm{~g} / \mathrm{kg}$ had no significant effect on DM intake post-weaning 4 weeks. Similarity, when Yang et al [28] fed the piglet liquid feed in a ratio of $1: 2$ from $\mathrm{d} 0$ to $\mathrm{d} 28$, no difference was found in ADFI, but there was an enhanced G:F. There are three possible reasons for this result. Firstly, water plays a crucial role in the muscle growth, which is a major part of the composition of organ and tissues, so enough water intake could be a reason for better G:F ratio. Secondly, a better transition from milk feeding can reduce the weaning stress and lead to a better G:F ratio. Thirdly, comparing with dry feed treatment, a lower fecal score and a tendency of better energy digestibility in wet feed group presented in this study also contributed to the improved G:F ratio.

Probiotics and nutrition density showed an interactive relationship in G:F ratio, which meant probiotics improved G:F ratio more dramatically in the high-nutrition diet. Similarly, our previous studies of Meng et al [29] and Yan et al [30] reported supplementation probiotics in high nutrition diets raised nutrient digestibility and reduced fecal gas emissions in growing pigs. And they believed that the interactive effect could be the increased microflora balance, which led to a better metabolism and transformation of feed into body mass. In our viewpoint, piglets fed relatively higher nutrition diet are more likely to suffer nutritional diarrhea, which results from that indigestible substrate inducing an explosive growth of bacteria and a disturbance of the colonization resistance [31]. Normally transient Escherichia coli strains in the gut [32] can multiply and attach. This study confirmed that the reason for positive effect in relatively high nutrition diet might be that probiotics play a role in balancing gut microflora, benefitting intestinal integrity to relieve intestinal stress under high nutrition [33]. However, Lan et al [34] whose paper reported the beneficial effects of probiotics complex supplementation on ADFI is more dramatic with low nutrient density $(3,850 \mathrm{kcal} / \mathrm{kg}$ vs $4,000 \mathrm{kcal} / \mathrm{kg})$, believed pigs were able to get same energy by increasing FI when low energy diets were provided. The difference interaction between nutrition level and probiotics might be caused by the actual energy in those trial diets and the different growth stages. There was a positive fecal score interaction between high nutrition diet and liquid feed. Relatively, the G:F ratio was improved more dramatically with liquid feed in the highnutrition diet. Those two results might imply that comparing to NRC [10] nutrition recommendation, a higher nutrition formula should be considered when liquid feed is fed to piglets. Interestingly, there was an interaction on G:F among nutrition levels, probiotics and liquid feed in phase 3. Therefore, supplementation probiotics into liquid diet at high nutrition might be a whole solution to improve growth performance and health status in post-weaning pigs. When piglets are fed in different feeding programs or at various nutrition designs, additives applications should be considered specifically. However, the interaction could not be found in overall growth performance.

\section{CONCLUSION}

These results indicated that probiotics in a supplementation diet could benefit growth performance (ADG, ADFI, and G:F) and reduce the frequency of watery feces. Besides, a wet feed program (feed:water $=1: 1.25$ ) could improve the G:F. Because there were two positive interactions: one between liquid program and nutrition density, the other between supplementation probiotics and nutrition density, the effect of liquid feed or probiotic could be influenced by dietary nutrition density in weaned piglets. An increasing value of G:F was 
obtained when wet feeding a high nutrition diet $(100 \mathrm{kcal}$ higher than NRC [10] recommendations) was supplemented with probiotics for 15 to 25 days.

\section{CONFLICT OF INTEREST}

We certify that there is no conflict of interest with any financial organization regarding the material discussed in the manuscript. Song Zhang is an employee of Kemin Industries (China) Co., Ltd. and Yoo DH is an employee of All The Best Co., Ltd..

\section{ACKNOWLEDGMENTS}

This work was carried out with the support of "Cooperative Research Program for Agriculture Science and Technology Development (Project No. PJ014485012020)" Rural Development Administration, Republic of Korea and was supported by the research fund of Dankook university in 2019 for the University Innovation Support Program.

\section{REFERENCES}

1. Lalle's JP, Paolo B, Hauke S, Stokes CR. Nutritional management of gut health in pigs around weaning. Proc Nutr Soc 2007;66:260-8. https://doi.org/10.1017/S0029665107005484

2. Barba-Vidal E, Castillejos L, López-Colom P, Urgell MR, Moreno Muñoz JA, Martín-Orúe SM. Evaluation of the probiotic strain Bifidobacterium longum subsp. infantis CECT 7210 capacities to improve health status and fight digestive pathogens in a piglet model. Front Microbiol 2017;8:533. https://doi.org/10.3389/fmicb.2017.00533

3. Shu Q, Qu F, Gill HS, et al. Probiotic treatment using Bifidobacterium lactis HN019 reduces weanling diarrhea associated with rotavirus and Escherichia coli infection in a piglet model. J Pediatr Gastroenterol Nutr 2001;33:171-7. https://doi.org/10. 1097/00005176-200108000-00014

4. Davis ME, Parrott T, Brown DC, et al. Effect of a Bacillusbased direct-fed microbial feed supplement on growth performance and pen cleaning characteristics of growing-finishing pigs. J Anim Sci 2008;86:1459-67. https://doi.org/10.2527/ jas.2007-0603

5. Chen YJ, Son KS, Min BJ, Cho JH, Kwon OS, Kim IH. Effects of dietary probiotic on growth performance, nutrients digestibility, blood characteristics and fecal noxious gas content in growing pigs. Asian-Australas J Anim Sci 2005;18:14648. https://doi.org/10.5713/ajas.2005.1464

6. Barba-Vidal E, Martin-orue SM, Catilejos L. Practical aspects of the use of probiotics in pig production: a review. Livest Sci 2019;233:84-96. https://doi.org/10.1016/j.livsci.2019.02.017

7. Meunier-Salaün MC, Chiron J, Etore F, et al. Review: Drinking water for liquid-fed pigs. Animal 2017;11:836-44. https://doi. org/10.1017/S1751731116002202
8. Missotten JAM, Michiels J, Ovyn A, De Smet S, Dierick NA. Fermented liquid feed for pigs. Arch Anim Nutr 2010;64: 437-66. https://doi.org/10.1080/1745039X.2010.512725

9. Russell PJ, Geary TM, Brooks PH, Campbell A. Performance, water use and effluent output of weaner pigs fed ad libitum with either dry pellets or liquid feed and the role of microbial activity in the liquid feed. J Sci Food Agric 1996;72:8-16. https:// doi.org/10.1002/(SICI)1097-0010(199609)72:1<8::AIDJSFA646>3.0.CO;2-K

10. National Research Council (NRC). Nutrient requirement of pigs, 10th edn. Washington, DC, USA: National Academy Press; 2012.

11. AOAC. Official methods of analysis. 19th ed. Washington, DC, USA: AOAC Int.; 2012.

12. European Commission. Commission Directive 98/64/EC of 3 September 1998 establishing Community methods of analysis for the determination of amino-acids, crude oils and fats, and olaquindox in feeding stuffs and amending Directive 71/393/EEC. Off J Eur Commun; 1998. pp. 14-28. Eur-Lex31998L0064

13. Hu CH, Gu LY, Luan ZS, Song J, Zhu K. Effects of montmorillonite-zinc oxide hybrid on performance, diarrhea, intestinal permeability and morphology of weanling pigs. Anim Feed Sci Technol 2012;177:108-15. https://doi.org/10.1016/j.ani feedsci.2012.07.028

14. SAS Institute. SAS user's guide: statistics. Version 7.0 ed. Cary, NC, USA: SAS Institute Inc.; 1998.

15 Lan RX, Lee SI, Kim IH. Effects of multistrain probiotics on growth performance, nutrient digestibility, blood profiles, faecal microbial shedding, faecal score and noxious gas emission in weaning pigs. J Anim Physiol Anim Nutr 2016; 11:1130-8. https://doi.org/10.1111/jpn.12501

16. Bhandari SK, Xu B, Nyachoti CM, Giesting DW, Krause DO. Evaluation of alternatives to antibiotics using an Escherichia coli $\mathrm{K} 8^{+}$model of piglet diarrhea: effects on gut microbial ecology. J Anim Sci 2008;86:836-47. https://doi.org/10.2527/ jas.2006-822

17. Gebru E, Lee JS, Son JC, et al. Effect of probiotic, bacteriophage, or organic acid-supplemented feeds or fermented soybean meal on the growth performance, acute-phase response, and bacterial shedding of grower pigs challenged with Salmonella enterica serotype Typhimurium. J Anim Sci 2010; 88:3880-6. https://doi.org/10.2527/jas.2010-2939

18. Davis ME, Parrott T, Brown DC, et al. Effect of a Bacillusbased direct-fed microbial feed supplement on growth performance and pen cleaning characteristics of growing-finishing pigs. J Anim Sci 2008;86:1459-67. https://doi.org/10.2527/ jas.2007-0603

19. Shu Q, Qu F, Gill H. Probiotic treatment using Bifidobacterium lactis HN019 reduces weanling diarrhea associated with rotavirus and Escherichia coli infection in a piglet model. J Pediatr Gastroenterol Nutr 2001;33:171-7. https://doi.org/10.1097/ 
00005176-200108000-00014

20. Taras D, Vahjen W, Macha M, Simon O. Response of performance characteristics and fecal consistency to long-lasting dietary supplementation with the probiotic strain Bacillus cereus var. toyoi to sows and piglets. Arch Anim Nutr 2005;59: 405-17.

21. Nguyen DH, Nyachoti CM, Kim IH. Evaluation of effect of probiotics mixture supplementation on growth performance, nutrient digestibility, faecal bacterial enumeration, and noxious gas emission in weaning pigs. Ital J Anim Sci 2019;18:466-73. https://doi.org/10.1080/1828051X.2018.1537726

22. Zhao PY, Kim IH. Effect of direct-fed microbial on growth performance, nutrient digestibility, fecal noxious gas emission, fecal microbial flora and diarrhea score in weanling pigs. Anim Feed Sci Technol 2015;200:86-92. https://doi.org/10.1016/j. anifeedsci.2014.12.010

23. Zijlstra RT, Whang KY, Easter RA, Odle J. Effect of feeding a milk replacer to early-weaned pigs on growth, body composition, and small intestinal morphology compared with suckled littermates. J Anim Sci 1996;74:2948-59. https://doi. org/10.2527/1996.74122948x

24. Mccracken BA, Spurlock ME, Roos MA, Zuckermann FA, Gaskins HR. Weaning anorexia may contribute to local inflammation in the piglet small intestine. J Nutr 1999;129: 613-9. https://doi.org/10.1093/jn/129.3.613

25. Kim JH, Heo KN, Odle J, Han K, Harrell RJ. Liquid diets accelerate the growth of early-weaned pigs and the effects are maintained to market weight. J Anim Sci 2001;79:42734. https://doi.org/10.2527/2001.792427x

26. Price KL, Lin X, Van Heugten E, Odle R, Willis G, Odle J. Diet physical form, fatty acid chain length, and emulsification alter fat utilization and growth of newly weaned pigs. J Anim Sci 2013;91:783-92. https://doi.org/10.2527/jas.2012-5307

27. Geary TM, Brooks PH, Morgan DT, Campbell A, Russell PJ. Performance of weaner pigs fed ad libitum with liquid feed at different dry matter concentrations. J Sci Food Agric 1996;
72:17-24. https://doi.org/10.1002/(SICI)1097-0010(199609) 72:1<17::AID-JSFA598>3.0.CO;2-3

28. Yang JS, Lee JH, Ko TG, et al. Effects of wet feeding of processed diets on performance, morphological changes in the small intestine and nutrient digestibility in weaned pigs. AsianAustralas J Anim Sci 2001;14:1308-15. https://doi.org/10.5713/ ajas.2001.1308

29. Meng QW, Yan L, Ao X, et al. Influence of probiotics in different energy and nutrient density diets on growth performance, nutrient digestibility, meat quality, and blood characteristics in growing-finishing pigs. J Anim Sci 2010;88:3320-6. https:// doi.org/10.2527/jas.2009-2308

30. Yan L, Kim IH. Effect of probiotics supplementation in diets with different nutrient densities on growth performance, nutrient digestibility, blood characteristics, faecal microbial population and faecal noxious gas content in growing pigs. J Appl Anim Res 2013;41:23-8. https://doi.org/10.1080/097 12119.2012.739092

31. Hopwood DE, Hampson DJ. Interactions between the intestinal microflora, diet and diarrhoea, and their influences on piglet health in the immediate post-weaning period. In: Pluske JR, Dividich J, Verstegen MWA, editors. Weaning the pig. Wageningen, The Netherlands: Wageningen Academic Publishers; 2003. pp. 199-218.

32. Nabuurs MJA. Etiology and pathogenic studies on post weaning diarrhea $[\mathrm{PhD}$ thesis]. Utrecht, The Netherlands: State University Utrecht; 1991.

33. Dong XL, Zhang NF, Zhou M, Tu Y, Deng K, Diao Q. Effects of dietary probiotics on growth performance, faecal microbiota and serum profiles in weaned piglets. Anim Prod Sci 2013;54:616-21. https://doi.org/10.1071/AN12372

34. Lan RX, Tran HN, Kim IH. Effects of probiotic supplementation in different nutrient density diets on growth performance, nutrient digestibility, blood profiles, fecal microflora and noxious gas emission in weaning pig. J Sci Food Agric 2017;97:1335-41. https://doi.org/10.1002/jsfa.7871 